\title{
Molecular diagnostics of neurodegenerative disorders
}

\author{
Megha Agrawal ${ }^{1 *}$ and Abhijit Biswas ${ }^{2}$ \\ ${ }^{1}$ Department of Biology, University of Arkansas at Little Rock, Little Rock, AR, USA, ${ }^{2}$ Department of Electrical Engineering, \\ Center for Nano Science and Technology, University of Notre Dame, Notre Dame, IN, USA
}

\section{OPEN ACCESS}

Edited by:

Weian Zhao,

University of California, Irvine, USA

Reviewed by:

Michal Mielcarek

Imperial College London, UK

Christopher E. Levy,

University of California, Irvine, USA

${ }^{*}$ Correspondence:

Megha Agrawal,

Department of Biology, University of Arkansas at Little Rock, 2801 South

University Avenue, Little Rock, AR 72204, USA

meghaagra@gmail.com;

mxagrawa/@ualr.edu

Specialty section:

This article was submitted to

Molecular Diagnostics,

a section of the journal

Frontiers in Molecular Biosciences

Received: 08 April 2015 Accepted: 04 September 2015

Published: 22 September 2015

Citation:

Agrawal M and Biswas A (2015)

Molecular diagnostics of neurodegenerative disorders.

Front. Mol. Biosci. 2:54. doi: 10.3389/fmolb.2015.00054
Molecular diagnostics provide a powerful method to detect and diagnose various neurological diseases such as Alzheimer's and Parkinson's disease. The confirmation of such diagnosis allows early detection and subsequent medical counseling that help specific patients to undergo clinically important drug trials. This provides a medical pathway to have better insight of neurogenesis and eventual cure of the neurodegenerative diseases. In this short review, we present recent advances in molecular diagnostics especially biomarkers and imaging spectroscopy for neurological diseases. We describe advances made in Alzheimer's disease (AD), Parkinson's disease (PD), Amyotrophic lateral sclerosis (ALS) and Huntington's disease (HD), and finally present a perspective on the future directions to provide a framework for further developments and refinements of molecular diagnostics to combat neurodegenerative disorders.

Keywords: biomarkers, miRNAs, Alzheimer's disease, Parkinson's disease, Amyotrophic lateral sclerosis, Huntington's disease, neurodegeneration, neurons

\section{Introduction}

Neurodegenerative disorders correspond to the disorders in the central nervous system that are characterized by the progressive loss of neural tissues. Changes in the neurons cause them to function abnormally and eventually result in the cells' demise. The reason is the inability of the neurons to regenerate on their own after the neural deterioration or severe damage. At present, roughly around 5 million Americans suffer from Alzheimer's disease (AD); 1 million from Parkinson's disease (PD); 400,000 from multiple sclerosis (MS); 30,000 from Amyotrophic lateral sclerosis (ALS), and 3000 from Huntington's disease (HD). The incidence is expected to soar as the population ages, because neurodegenerative diseases strike primarily in mid-to late-life. Neuroregeneration is a viable way to curb neurodenegerative disorders. One of the current approaches is stem cell therapy that has shown to be potentially helpful in neuroregeneration or even neuronal cell replacement (Chung et al., 2002; Rachakonda et al., 2004).

An early detection of the onset of neurodegeneration is vital as it can provide a chance for an early treatment that may be helpful to prevent further progression of the disease. Among current diagnostics, neuropathology is considered as the gold standard (Chung et al., 2002). However, it is usually based on an autopsy that is done after the death of a patient. Therefore, medical researchers are in search for an effective non-invasive diagnostic method that can be employed for an early detection of neurodegeneration when a pharmacological intervention is still possible.

Molecular diagnosis has emerged as a powerful technique that can be helpful for an early detection of various neurodegenerative disorders. One of the powerful molecular diagnostics is the application of biomarkers. Biomarkers are basically biological molecular substances that are used to indicate the presence or onset of a certain disorder. Normal and abnormal biological processes 
can be detected by the use of biomarkers. The principal requirement for a good biomarker is its preciseness and reliability. It should also be able to distinguish between the healthy and the diseased tissues, and should differentiate between different diseases. Biomarkers are considered promising in aiding in early diagnosis and setting standards for the development of new remedies to treat neuronal disorders (Chung et al., 2002; Peterson, 2002; Rachakonda et al., 2004). Biomarkers may be measured using imaging techniques such as positron emission tomography (PET), magnetic resonance imaging (MRI), and nuclear magnetic resonance spectroscopy (NMRS). Table 1 summarizes various molecular diagnostic markers for neurodegenerative diseases.

Among other recently developed molecular diagnostics, biomarkers coupled with magnetic resonance spectroscopy (MRS) has shown promise. It is a quantitative imaging technique that allows in-vivo measurement of certain neuronal metabolites as biomarkers that can be used to study metabolic dysfunctions

TABLE 1 | A summary giving genetic and biochemical diagnostic markers for Alzheimer's, Parkinson's, Amyotrophic lateral sclerosis, and Huntington's Disease.

\begin{tabular}{|c|c|c|}
\hline Diseases & Genetic diagnostic markers & $\begin{array}{l}\text { Biochemical } \\
\text { diagnostic } \\
\text { markers }\end{array}$ \\
\hline \multirow[t]{5}{*}{$\begin{array}{l}\text { Alzheimer's } \\
\text { Disease (AD) }\end{array}$} & Amyloid precursor protein mutations & $\begin{array}{l}\text { Plasma/CSF } \\
\text { A } \beta_{1-42} \text { peptide }\end{array}$ \\
\hline & Presinilin-1 gene mutations & CSF tau protein \\
\hline & Presinilin-2 gene mutations & Phospho-tau \\
\hline & ApoE isoforms & \\
\hline & ApoEpolymormisms & \\
\hline \multirow[t]{6}{*}{$\begin{array}{l}\text { Parkinson's } \\
\text { Disease (PD) }\end{array}$} & $\alpha$-synuclein gene mutations & $\begin{array}{l}\text { Loss of Dopamine } \\
\text { transporter (DAT) }\end{array}$ \\
\hline & Parkin gene mutations & Lewy bodies \\
\hline & $U C H-L 1$ gene mutations & \\
\hline & PINK1 gene mutations & \\
\hline & DJ-1 gene mutations & \\
\hline & NR4A2 gene mutations & \\
\hline \multirow{6}{*}{$\begin{array}{l}\text { Amyotrophic } \\
\text { Lateral Sclerosis } \\
\text { (ALS) }\end{array}$} & ALS2 gene mutations & mGLUR2 \\
\hline & NEFH gene mutations & SOD1 \\
\hline & SOD1 gene mutation & Glutathione \\
\hline & C9orf72 gene mutation & $8 \mathrm{OH} 2^{\prime} \mathrm{dG}$ \\
\hline & FUS gene mutation & Cytokines \\
\hline & TARDBP gene mutations & \\
\hline \multirow{6}{*}{$\begin{array}{l}\text { Huntington's } \\
\text { Disease (HD) }\end{array}$} & HTT gene mutations & Growth hormones \\
\hline & & Cytokines \\
\hline & & mGLUR2 \\
\hline & & SOD1 \\
\hline & & Glutathione \\
\hline & & $80 H 2^{\prime} d G$ \\
\hline
\end{tabular}

and irreversible neuronal damage (Ciurleo et al., 2014). The potential role of MRS as an in-vivo molecular imaging biomarker was investigated for early diagnosis of PD and for monitoring the efficacy of therapeutic interventions (Ciurleo et al., 2014).

Altered expression of microRNAs (miRNAs) in many disease states, including neurodegeneration along with applications of miRNAs in biological fluids in different pathologies make them promising candidates as neurodegenerative disease biomarkers that may lead to identify new therapeutic targets (Grasso et al., 2014). The role of miRNAs in the pathogenesis of neurodegeneration was investigated to gain insights into the possibilities and challenges of using these small RNA molecules as a signature for neurodegenerative conditions (Grasso et al., 2014). It is known that miRNAs can be transported by exosomes which are small membrane-derived vesicles secreted by many cell types, including neurons, astrocytes, oligodendrocytes, and microglia (Lachenal et al., 2011; Russo et al., 2012). Exosomes shed from stimulated blood cells and the vascular endothelium are involved in neurological disorders (Soreq et al., 2013). Exosomes have significant potential as biomarkers for disease diagnosis, as their molecular composition reflects the physiological or pathophysiological changes in their cell of origin (Lin et al., 2015). Furthermore, they can be isolated from biofluids such as blood and urine, making them very attractive targets for diagnostic application. It has been reported that exosomal amyloid peptides accumulate in the brain plaques of $\mathrm{AD}$ patients (Rajendran et al., 2006) and tau phosphorylated at Thr-181, an established biomarker for $\mathrm{AD}$, is present at elevated levels in exosomes isolated from cerebrospinal fluid specimens of $\mathrm{AD}$ patients with mild symptoms (Saman et al., 2012). Studies have also shown release of $\alpha$-synuclein in exosomes in an in vitro model system of PD (Alvarez-Erviti et al., 2011). These exosomal proteins may have great potential in clinical diagnostics and should be further explored, as the concept is still new in the biomarker discovery arena (Miller and O'Callaghan, 2015).

The research field of molecular diagnostics in neurodegenerative disorder is still a nascent area of research and development. It is anticipated that further developments in various molecular diagnostics would pave the way for the early detection and effective treatment of neurodegeneration. In this short review, we focus on recent advances made in major neurodegenerative disorders- $\mathrm{AD}, \mathrm{PD}, \mathrm{ALS}$, and $\mathrm{HD}$ and the applications of molecular diagnosis for the potential remedies. We also present a perspective on the future direction of diagnostics and curbing the progression of neuronal disorders.

\section{Molecular Diagnosis for Neurodegenerative Diseases: A Potentially Better Approach Over Behavioral Symptoms Based Diagnosis}

Behavioral symptoms can be utilized for the pre-mortem diagnosis of neurodegenerative disorders. However, the major drawback of behavioral symptoms based diagnosis is its limitations to identify patients early in the course of their disease, when the pharmacological intervention can significantly prevent 
further progression of the disease, if detected early. For example, well-established behavioral tests like the ADAS-Cog that are regarded as the "gold-standard" for AD diagnosis may give falsenegative results for patients with mild symptoms (Posner et al., 2013).

To overcome these diagnostics challenges, current neuropathologic methods have been combined with molecular biology techniques that have led to increased understanding of neurodegenerative disorders along with biologically based classifications of these disorders. Molecular diagnostics provide a powerful tool in the diagnosis of many neurological diseases. For example, genetic testing of mutations in disease-causinggenes has been leveraged to define and classify many of the heterogeneous inherited neurodegenerative syndromes (Gasser et al., 2001a,b, 2003). Changes in pathologies, biochemistries and genetics of patients can give us comprehensive information regarding the nature of a particular disease. However, molecular testing may be performed only after careful consideration and a genetic counseling. We describe in the following sections major neurologic disorders, and the ability and applications of molecular diagnostics in their detection.

\section{Alzheimer's Disease}

$\mathrm{AD}$ is the most common neurodegenerative disease in most Countries; it is a progressive, degenerative disorder that attacks the brain's neurons, resulting in loss of memory, thinking and language skills, and behavioral changes. Most of the neurons that degenerate in this disease communicate with other neurons using the chemical or neurotransmitter acetylcholine in the brain. Alterations in acetylcholinesterase (AChE) and butyrylcholinesterase $(\mathrm{BuChE})$ can be observed in $\mathrm{AD}$ but acetylcholine depletion is the most common and striking of all symptoms (Rachakonda et al., 2004).

The two types of abnormal lesions that clog the brains of Alzheimer's patients are extracellular senile plaques (composed of beta-amyloid peptides) and intracellular neurofibrillary tangles (NFTs, composed of tau protein). A $\beta$ forms as a result of enzymatic cleavage of the parent Amyloid Precursor Protein (APP). Proteases that are involved in the breakdown of APP, are $\alpha-, \beta-$, and $\gamma$ - secretases (Hardy and Selkoe, 2002; Rachakonda et al., 2004). The NFTs account for the synaptic degeneration or the atrophy of nerve cells following damage to the synaptically connected axons. NFTs are composed of paired helical filaments (PHF), which are principally made up of hyperphosphorylated insoluble form of tau protein (el-Agnaf and Irvine, 2002).

Diagnosis of $\mathrm{AD}$ is usually based on clinical observations and cognitive testing like neuropsychological testing which helps in the diagnosis and treatment of conditions like $\mathrm{AD}$ that affect emotion, thinking and behavior (Harvey, 2012). Neuropsychological tests accompany a comprehensive interview with the patient, and include tests to assess attention, memory, language, the ability to plan and reason, and modify behavior, as well as assessments of personality and emotional stability, that can also help the doctor and family better understand the impact of a disorder on a patient's everyday functioning. The disease is eventually confirmed by postmortem by demonstrating amyloid plaques and neurofibrillary tangles in the brain. However, the progression of the disease process is an ongoing phenomenon that significantly damages the brain long before clinical findings appear. Hence, molecular biologists, biomedical, and medicine experts and biotechnologists are coming together to design and develop advanced diagnostic molecular markers that might allow very early-stage diagnosis of $\mathrm{AD}$ and the objective assessment of its responses to putative treatments (Rachakonda et al., 2004). Several genetic and biochemical diagnostic biomarkers have been employed to detect and diagnose AD.

\section{Genetic Biomarkers}

Less than $5 \%$ of all cases of $\mathrm{AD}$ can be accounted for by mutations in the following three genes. Amongst them, mutations on the two homologous presenilin genes: presenilin 1 (PS1, MIM 104 311) located on chromosome 14, and presenilin 2 (PS2, MIM 600 759) located on chromosome 1, are most common and are responsible for over half of the known familial $\mathrm{AD}$ cases, whereas mutations in the gene for amyloid precursor protein (APP, MIM 104 760) located on chromosome 21) are comparatively less (Gasser et al., 2001a; Rachakonda et al., 2004). The presenilin genes code for proteins known as presenilin, which control the APP proteolysis into smaller peptides (Goodall et al., 2013). An abnormal increase in the activity of APP can be due to any missense mutation on one of these presenilin genes resulting into more $A \beta$ peptides (Berezovska et al., 2003). The first genetic mutation linked to $\mathrm{AD}$ was found on the $\beta A P P$ gene (Rachakonda et al., 2004). This $\beta A P P$ gene, encodes a glycosylated trans-membrane protein which contains 770 amino acids in its longest isoform. This was confirmed by the fact that patients with Down's syndrome also developed similar plaques and suffered Alzheimer encephalopathy in their later years (Rachakonda et al., 2004). In addition to the mutations mentioned above, which can cause AD, the E4 allele of the ApoE is associated with the sporadic forms of $\mathrm{AD}$ (Bekris et al., 2010). Although E4 allele was detected in about $40-50 \%$ of all AD patients, but could not serve as a diagnostic marker based on the sensitivity criteria for biomarkers. Therefore, ApoE is regarded as a risk factor indicator rather than an actual genetic marker of AD. Along with positive family history, an early onset (in the 40s and 50s) which is common to all these monogenic forms, should act as an indication for molecular genetic diagnosis.

\section{Biochemical Markers}

The levels of tau protein and $\mathrm{A} \beta$ in cerebrospinal fluid (CSF) are the two most promising biochemical markers of $\mathrm{AD}$. $\mathrm{A} \beta$ is secreted into the extracellular space and biological fluids, including CSF making $\mathrm{A} \beta 42$ a considerable indicator of $\mathrm{AD}$ (Sunderland et al., 2003). A decrease in levels of A $\beta$ in CSF reflects $\mathrm{AD}$ and its sensitivity is around $80-90 \%$. As $\mathrm{AD}$ progresses $A \beta$ peptide from CSF aggregates to form plaques in the brain, thereby, lowering its concentration in the CSF. CSF-A $\beta 42$ appears to be a remarkable biomarker for diagnosis of $\mathrm{AD}$ when used in combination with other $\mathrm{AD}$ biomarkers. CSF-tau also provides a very high sensitivity for $\mathrm{AD}$ but the reason for its abnormal increase in $\mathrm{AD}$ patients is not clear. The combination of both CSF-A $\beta 42$ and CSF-tau may 
improve their specificity and sensitivity and can be an ideal biochemical marker set for AD (Sjögren et al., 2003). 2-(1-\{6$\left[\left(2-\left[{ }^{18} \mathrm{~F}\right]\right.\right.$ Fluoroethyl) (methyl)amino]-2-naphthyl\}ethylidene) malononitrile ( $\left.\left({ }^{18} \mathrm{~F}\right] \mathrm{FDDNP}\right)-\mathrm{PET}$ can determine the localization and load of neurofibrillary tangles and senile amyloid plaques in the human brain because of its ability to cross the Blood-BrainBarrier (BBB). FDG-PET is used to measure the brain's energy utilization and to infer synaptic number (Wurtman, 2015). Therefore, PET Molecular imaging is employed to diagnose AD (Rachakonda et al., 2004; Sair et al., 2004). This discovery of a new binding site to $A \beta_{40}$ fibrils as a result of FDDNP binding provides a new opportunity for early treatment of $\mathrm{AD}$. The clinical sensitivity for $\mathrm{AD}$ using the "probable $\mathrm{AD}$ " category is $66 \pm 17 \%$ relative to neuropathologically conformed diagnoses and can be increased to $90.5 \pm 5.5 \%$, by including "possible $\mathrm{AD}$ " patients at the expense of specificity. The sensitivity of $18 \mathrm{~F}-$ FDG PET is $91 \pm 3 \%$ (Bokde et al., 2011). The combined use of the $\left[{ }^{18} \mathrm{~F}\right]$ FDDNP-PET molecular diagnostic labeling system and other diagnostic tests provide a new pathway to early diagnosis of AD (Sair et al., 2004; Wurtman, 2015). In a related work employing imaging spectroscopy, SPECT and PET tracers were used in the diagnosis and investigation of $\mathrm{AD}$. Most tracers demonstrate the neuronal loss associated with the condition and results in regional decrease in glucose utilization which can be studied with ${ }^{18} \mathrm{~F}$-FDG PET imaging (Young, 2009). SPECT and PET imaging are far more sensitive with the ability to detect tracers at $10^{-9}-10^{-11} \mathrm{~mol} / \mathrm{L}$, at concentrations of tracers that will not disturb normal function (Bokde et al., 2011).

MicroRNAs (miRNAs) have been employed for early detection of AD (Grasso et al., 2014). miRNAs belong to a family of short, single-stranded 21-22 nucleotides-long non-coding RNAs that constitute about $1 \%$ of all human genes. They represent the most abundant class of small RNAs in animals. Further, miRNAs are found in high abundance within the nervous system, where they often replicate a brain-specific expression pattern and are usually found to be co-expressed with their targets. Their main roles are as key regulators of different biological functions including synaptic plasticity and neurogenesis, where they channelize the cellular physiology toward neuronal differentiation. Also, they can indirectly influence neurogenesis by regulating the proliferation and self-renewal of neural stem cells (Grasso et al., 2014).

It is interesting to note that miRNAs are deregulated in several neurodegenerative diseases, a spectrum of etiologies culminating in a final common pathway of neuronal cell death (Goodall et al., 2013). The dysfunction of miRNAs in neurodegenerative disorders can be leveraged for early diagnosis of $\mathrm{AD}$, which is a novel approach to understanding neurodegenerative diseases (Goodall et al., 2013). Further, the use of miRNAs as biomolecular diagnostics markers has some advantages: first of all they allow ease of detection with extreme specificity. Furthermore, unlike large RNA molecules as mRNAs, miRNAs can be well preserved in formalin, paraffin embedded tissues (FFPE) and also in fresh snap-frozen specimens (Xi et al., 2007; Grasso et al., 2014).

Mild cognitive Impairment (MCI) is an intermediate state between normal aging and $\mathrm{AD}$ (and other dementias), which is usually defined as the first stage when clinical symptoms become evident (DeCarli, 2003). Plasma miRNA biomarkers were reported to detect MCI, where an initial pool of miRNAs was selected among known brain- and neuron-enriched miRNAs (Sheinerman et al., 2012). The researchers then identified biomolecular diagnostic marker pairs represented by two sets: the "miR-132 family" that consist of $\mathrm{miR}-128 / \mathrm{miR}-491-5 \mathrm{p}$, $\mathrm{miR}-132 / \mathrm{miR}-491-5 \mathrm{p}$ and $\mathrm{miR}-874 / \mathrm{miR}-491-5 \mathrm{p}$ and the "miR-134 family" comprising $\mathrm{miR}-134 / \mathrm{miR}-370$, $\mathrm{miR}-323-3 \mathrm{p} / \mathrm{miR}-370$ and $\mathrm{miR}-382 / \mathrm{miR}-370$ with fairly high sensitivity and specificity at $79-100 \%$ and $79-95 \%$, respectively. In a separate longitudinal study, the identified miRNA biomolecular diagnostic marker pairs successfully detected MCI in majority of patients at asymptomatic stage 1-5 years prior to clinical diagnosis (Sheinerman et al., 2012; Grasso et al., 2014).

Recent observations were made related to change in the levels of plasma phospholipids; that can be leveraged for developing new bimolecular diagnostic marker for AD. The reduction in the level of phospholipids is anticipated to enable the accurate prediction, that whether a cognitively normal individual is going to develop MCI or AD within 2 years (Wurtman, 2015). There is one study on blood-based biomarker panel for detecting preclinical $\mathrm{AD}$ with above $90 \%$ accuracy greater than that obtained from most published CSF studies (Mapstone et al., 2014).

\section{Parkinson's Disease}

The search for molecular diagnostics biomarkers in PD is critical to identify the disease in early stages which will allow monitoring the effectiveness of neuroprotective therapies for PD (Molochnikov et al., 2012). In PD, degeneration of neurons, more specifically dopaminergic neurons between the substantianigra (SN) and the striatum occur. As a result, a great majority of dopamine producing cells in the substantianigra are lost in patients with $\mathrm{PD}$. The symptoms of PD are trembling in hands, arms, legs, and face; stiffness of the limbs and trunk; slowness of movement; and impaired balance and coordination. As these neurons are progressively destroyed, patients may have difficulty walking, talking and completing other simple tasks (Rachakonda et al., 2004). PD usually affects people over the age of sixty.

Currently, only clinical criteria are employed to diagnose PD (Molochnikov et al., 2012). The evaluation of the clinical status and evolution of PD are based on various factors and medical steps. These include examination of symptoms, utilizing structured scoring systems [Unified Parkinson's Disease Rating Scale, (UPDRS), Short Parkinson Evaluation Scale, (SPES), Scales for Outcomes in Parkinson's diseases(SCOPA), and the Hoehn and Yahr (H\&Y) staging scale; (Molochnikov et al., 2012)]. Clinical criteria based diagnosis of PD can be done with a typical presentation and positive response to levodopa with a sensitivity of $93 \%$. However, the major limitation of this technique is differential diagnosis from other entities presenting Parkinsonism [e.g., essential tremor, progressive supranuclear palsy (PSP), multisystem 
atrophy (MSA), corticobasal degeneration (CBD)] that may be challenging.

Recent research has shown that molecular diagnostic tools can be leveraged to overcome the current challenges on limitations to early detection and effective differential diagnosis. A molecular diagnostic signature in blood that identifies early PD was reported. An assessment was done on whether a gene signature could be detected in blood from early/mild PD patients that could support the diagnosis of early PD, focusing on genes found particularly altered in the substantianigra of sporadic PD (Molochnikov et al., 2012). The research findings provide evidence on the ability of a five-gene panel to diagnose early/mild PD, with a possible diagnostic value for detection of asymptomatic PD before overt expression of the disorder (Molochnikov et al., 2012).

This pilot study demonstrated that the blood gene model can have strong predictive value for PD diagnosis that possibly may help to identify individuals at presymptomatic stages (patients with depression, sleep disturbances or hyposmia or patients carrying genetic risk factors) that are good candidates for neuroprotective treatment. Such a biomolecular diagnostic marker for PD can be of tremendous value for the identification of a pathophysiological subgroup of PD patients that may respond favorably to agents targeting the mechanisms reflected by the gene panel.

\section{Genetic Biomarkers}

$\mathrm{PD}$ is inherited in a Mendelian autosomal dominant or autosomal recessive fashion in a small number of families. Mutations were found in $\alpha$-synuclein (SNCA) and leucine-rich repeat kinase2 (LRRK2) genes for late-onset disease and parkin (PARK2), ubiquitin carboxy-terminal hydrolase L1 (UCH-L1), PTEN Induced Putative Kinase1 (PINK1), oncogene DJ1 (DJ1) for early onset (Grasso et al., 2014).

Point mutations, duplications, and triplication in the $\alpha$ synuclein gene, which is located on chromosome 4, are a characteristic of PD and they occur in most forms including the rare early onset familial form of PD. Genes and gene products have been identified by characterizing the monogenetic autosomal dominant forms of PD. Several gene products of the mutated genes in the autosomal dominant forms have been linked to mitochondrial dysfunction, oxidative stress, and mishandling of impaired or aberrant forms of the gene products (e.g., oligometric $\alpha$-synuclein) (Miller and O'Callaghan, 2015). More than 70 mutations on the large parkin gene have been associated with the early-onset form of Parkinsonism. Mutations in the parkin gene may account for PD in as many as 50\% of familial cases of autosomal recessive juvenile Parkinsonism (Pankratz et al., 2003). Another gene ubiquitin carboxy-terminal hydrolase L1 (UCH-L1) located on chromosome 4 encodes a protein which belongs to the family of deubiquitinating enzymes. Protein UCH-L1 constitutes $1 \%$ of brain protein and its function is presumed to act to recycle ubiquitin by hydrolyzing the ubiquitinated peptides. This enzyme plays a role in modifying the damaged proteins that might otherwise accumulate to toxic levels in the neuron (Leroy et al., 1998). Also two homozygous mutations in PINK1 gene associated with PD were found in Spanish and Italian families. This finding provided additional evidence that PINK1 mutations are associated with PARK6 (Valente et al., 2004). And the mutations associated with PARK7 are in DJ-1 gene (Bonifati et al., 2003). Evidence suggests DJ-1 protein involvement in oxidative stress and neurodegeneration. Slow progression of symptoms with sustained response to levodopa treatment is the clinical characteristics of DJ-1 Parkinsonism (Dekker et al., 2003). Revealing the physiological role of these genes may promote the understanding of the mechanisms of brain neuronal maintenance.

\section{Biochemical Markers}

Two other major biomolecular diagnostic markers have been employed to recognize the onset of PD. They include (1) the loss of the dopamine transporter "DAT" detected by PET imaging and (2) the presence of the $\alpha$-synuclein protein located in the Lewy body lesions. DAT mediates uptake of dopamine (DA) into dopaminergic neurons by an electrogenic, $\mathrm{Na}^{+}$- and $\mathrm{Cl}^{-}$transport-coupled mechanism. DA and cocaine (uptake blockers) would bind to both the shared and separate domains on the transporter, which is observed to be dramatically influenced by the presence of cations. DAT is also involved in the uptake of toxins generating Parkinson's syndrome. Thus, the localization of striatal, preferentially putamen DAT concentration is considered a high sensitivity parameter for the detection of early phases of PD and best molecular diagnostic marker (Marek et al., 2000; Rachakonda et al., 2004; Shinto et al., 2014). Discovery of Lewy bodies and Lewy neuritis, the characteristic lesions in brains of patients with PD and dementia is due to two mutations in $\alpha$-synuclein gene. Given that $\alpha$-synuclein is also found in other synucleinopathies, it should be used with the aid of other diagnostic methods to increase the specificity and sensitivity for PD (Duyckaerts and Hauw, 2003).

Several imaging techniques have been employed for the diagnosis of PD, for example, PET with $\left[{ }^{18} \mathrm{~F}\right]$-Dopa tracer (Loane and Politis, 2011) and single photon emission tomography (SPECT) with [123I]- $\beta$-CIT (Tissingh et al., 1997). PET is considered to be the most useful tool for PD diagnosis by measuring the emission of positrons from the brain after a small amount of radioactive isotopes or tracers have been injected into the blood stream. Studies have shown a mean reduction of $40 \%$ in striatal 18F-Dopa uptake between controls and patients with PD (Bokde et al., 2011). PD patients related to medication typically have normal $18 \mathrm{~F}$-Dopa distribution. Tracer $\left[{ }^{18} \mathrm{~F}\right]$-Dopa has very limited clinical availability but Ioflupane Iodine-123 (DatSCAN) is a widely available SPECT tracer which models the presynaptic dopamine receptor (DaT) system. SPECT only differ to PET in that it uses isotopes with longer half-lives that can be stored on site (Rachakonda et al., 2004; Young, 2009). Recently a SPECT imaging with ${ }^{99 \mathrm{~m}}$ Tc-TRODAT-1 was conducted in 16 consecutive PD patients ( 9 men; 7 women) and in 6 age matched healthy volunteers ( 4 men; 2 women; Shinto et al., 2014). The images were obtained $3 \mathrm{~h}$ after the intra-venous injection of the tracer. A stepwise reduction in specific striatal uptake of ${ }^{99 m}$ Tc-TRODAT-1 was found with increasing disease severity between healthy controls vs. Stage 
I vs. Stage II vs. Stage III in PD patients (i.e., 3.77 vs. 2.56 vs. 1.57 vs. $0.63, P<0.05)$. ${ }^{99} \mathrm{~m}$ Tc-TRODAT- 1 is accurate and widely available for the assessment of DAT activity. These techniques could improve differential diagnosis of Parkinsonism, but cost-effectiveness remains a problem (Jankovic et al., 2000; Molochnikov et al., 2012).

Biological fluids are excellent source for biomarkers as their close proximity to cells reflects their biological condition and are simple to obtain and cost-effective (Shinde et al., 2015). With the increasing relevance of miRNAs in biofluids the development of circulating biomarkers for PD has great potential. A study using qRT-PCR suggested that in peripheral blood the expression levels of miR-1, miR-22-5p, and miR-29 allow to distinguish PD patients from healthy subjects, and also miR-162-3p, miR-26a-2-3p, and miR30a differentiate between treated and untreated patients (Margis et al., 2011). In a recent study using next generation sequencing for total blood leukocytes it was found that, 16 miRNAs including miR-16, miE-20a and miR-320 significantly altered in PD patients compared to healthy controls (Soreq et al., 2013; Grasso et al., 2014).

There is no standard diagnostic test for Parkinson's. Researchers are still working to develop an accurate "biological marker," such as a blood test or an imaging scan. To date, tests consist of specialized brain scanning techniques to measure the dopamine system and brain metabolism is the best objective test for PD (Torrent et al., 2015). But these tests are expensive and performed only in specialized imaging centers.

\section{Amyotrophic Lateral Sclerosis}

ALS is a rapidly progressive, invariably fatal neurological disease that attacks the neurons responsible for controlling voluntary muscles. Messages from motor neurons in the brain (called upper motor neurons) are transmitted to motor neurons in the spinal cord (called lower motor neurons) and from them to particular muscles. In ALS, both the upper and lower motor neurons degenerate or die, and stop sending messages to muscles. Unable to function, the muscles gradually weaken, waste away (atrophy), and have very fine twitches (called fasciculation). Eventually, the ability of the brain to start and control voluntary movement is lost. ALS is a result of complex array of factors, including all or just some of these like oxidative stress, endoplasmic reticulum stress, mitochondrial dysfunction, dysregulated endosomal trafficking, dysregulated transcription, and RNA processing, excitotoxicity, apoptosis, inflammation, and genetic susceptibility (Figure 1; Calvo et al., 2014).

The diagnosis of ALS is primarily based on the symptoms, clinical examination, and a series of tests to rule out other diseases. We do not have a practical diagnostic biomarker for ALS in spite of intensive research over the past several years, which lead to diagnostic delays. Neurophysiological approaches like motor unit number estimation (MUNE) and electromyography (EMG), a special recording technique that detects electrical activity in muscles, routinely play a key role in search of valid biomarkers to recognize ALS (DeJesus-Hernandez et al., 2011; Joyce and Carter, 2013).

\section{Genetic Biomarkers}

Mutations in more than a dozen genes have been found to cause familial ALS. About one-third of all familial cases (and a small percentage of sporadic cases) results from a defect in a gene known as "chromosome 9 open reading frame 72," or C9orf72. The function of this gene is still unknown. Another 20 percent of familial cases result from mutations in the gene SOD1 that encodes the enzyme copper-zinc superoxide dismutase 1 (SOD1). Although it is still not clear how mutations in the SOD1 gene lead to motor neuron degeneration, there is increasing evidence that mutant SOD1 protein can become toxic. Another 5 percent of familial cases and $<1 \%$ sporadic cases results from mutations in the gene FUS, which encodes fused in sarcoma/translocated in liposarcoma (FUS/TLS), and three percent of the remaining familial cases result from mutations in TARDBP gene, which encodes TAR DNA binding protein-43 (TDP-43; Robelin and Gonzalez De Aguilar, 2014).

Another possibility is the role of epigenetics. Epigenetic mechanisms modify chromatin structure and can mimic genetic change; these modifications are reversible, heritable, and nonheritable in DNA or chromatin structure, but not in DNA sequence (Martin and Wong, 2013). There are different types of epigenetic modifications, such as DNA methylation and histone acetylation. In a study, CpG methylation microarrays were used to profile DNA extracts of postmortem spinal cord from sporadic ALS cases (Figueroa-Romero et al., 2012). Bisulfiteconverted DNA was amplified and hybridized to Infinium Human Methylation27 DNA BeadChip arrays. Methylation of 27,578 CpG sites spanning 14,495 human genes was determined and hypo- or hypermethylation 726 Martin and Wong was found in 112 genes in ALS cases (Figueroa-Romero et al., 2012).

\section{Biochemical Markers}

Studies showed that the expression of the metabotropic glutamate receptor subtype mGLUR2, which is known to provide protection against excitotoxicity, was diminished in ALS T lymphocytes (Poulopoulou et al., 2005), which was confirmed by the high concentration of glutamate detected in the CSF of many patients. The enzymatic activity of glutathione peroxidase and SOD1 was also found to decrease in ALS erythrocytes (Cova et al., 2010). Increased amounts of 8 -hydroxy- $2^{\prime}$-deoxyguanosine $\left(8 \mathrm{OH} 2^{\prime} \mathrm{dG}\right.$ in ALS patients, which is a product of the oxidative injury to DNA is also a good biomarker (Bogdanov et al., 2000). There is an uncontrolled increase of microglial cells in the central nervous system and other immune cells, contributing to motor neuron degeneration in ALS (Philips and Robberecht, 2011). The factors involved in these inflammatory reactions like cytokines can be followed in the periphery as potential biomarkers. Therefore, the circulating levels of interleukin-6, tumor necrosis factor- $\alpha$ (TNF$\alpha$ ), interferon- $\gamma$, monocyte chemo attractant protein-1 (MCP-1), and wide-range $\mathrm{C}$-reactive protein (wrCRP) were found to be increased in ALS patients (Robelin and Gonzalez De Aguilar, 2014). Neurite outgrowth inhibitor (Nogo), one of the potential biomarkers for ALS possesses axonal growth inhibitory activity and has a central role in ALS (Fergani et al., 2005). Together with the absence of reliable and powerful diagnostic and prognostic biomarkers, ALS is a major cause for concern. 


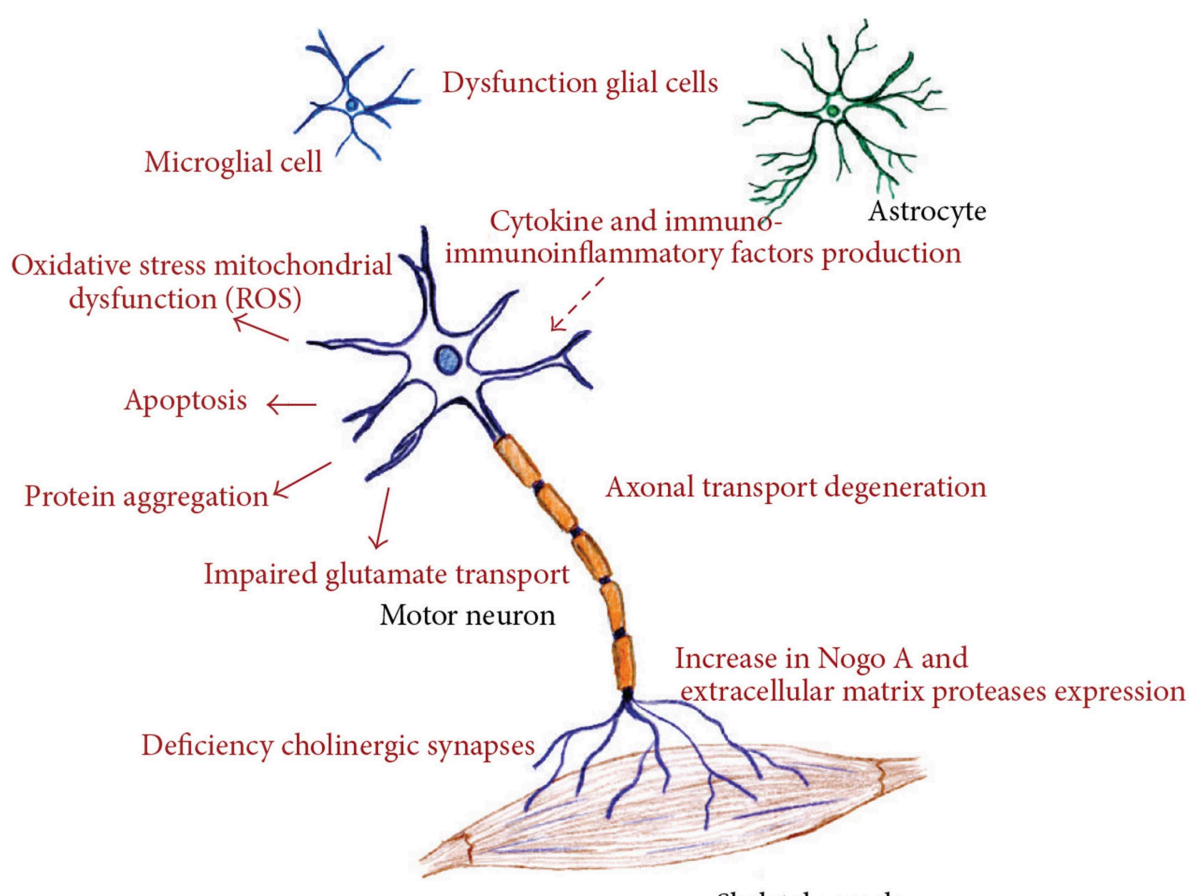

Skeletal muscle

FIGURE 1 | Different molecular pathways altered in amyotrophic lateral sclerosis, all of these deregulated mechanisms prompt motor neuron death (Figure taken from: Calvo et al., 2014).

\section{Huntington's Disease}

HD is a heritable neurodegenerative disorder that can affect motor, cognitive and psychiatric functioning. Decline of cognitive ability and change in personality are symptoms of HD (Mastrokolias et al., 2015). The pathology is caused by an expanded CAG repeat in the HTT gene, resulting in a mutant huntingtin protein (mHTT). Mutant protein aggregate formation and neuronal cell loss, with transcriptional deregulation are prominent feature of HD brain tissue (Runne et al., 2008). Recently, mutant huntingtin protein (mHTT) levels were quantified by an ultrasensitive single-molecule counting (SMC) mHTT immunoassay for the first time in CSF samples of individuals bearing $\mathrm{HD}$ mutation (Wild et al., 2015). It is important to have a disease progression biomarker that should be able to identify changes before clinical symptoms. Huntingtin is ubiquitously expressed and mutant huntingtin-specific changes could be reflected by gene expression changes in blood. Involvement of leukocytes in immune system regulation made blood an ideal source for identifying HD events such as peripheral inflammation. Several studies have also identified HD blood mRNA changes using microarray technology, but were difficult to validate across studies (Lovrecic et al., 2009). The validation of biomarkers for HD has been always challenging as the disease present itself through a variety of symptoms and progression rates.

\section{Molecular Biomarkers}

Individuals with $\mathrm{HD}$ over expressed the gene, H2A histone family, member Y (H2AFY), in their blood (Hu et al., 2011). The overexpression of this gene in both the blood and the brain was validated in samples from clinical studies. Specifically, the research demonstrates a 1.6 -fold overexpression of $H 2 A F Y$ in patients with HD. Recently a study showed gene expression profiling, with the help of next-generation sequencing and Fluidigm technologies and yielded a set of five genes as a potential HD biomarkers that are highly expressed in HD blood (Mastrokolias et al., 2015). Prokineticin 2 (PROK2) has been proposed to have a role in the circadian rhythms alterations that have been shown to correlate with cognitive impairment in HD (Aziz et al., 2010). Pharmacological imposition of sleep slows cognitive decline and reverses deregulation of PROK2 in HD models. $P R O K 2$ is very promising biomarker of $\mathrm{HD}$ progression. Evidences suggest that gene repression mechanisms are also associated with HD and Zinc finger protein 238 (ZNF238) is a transcriptional repressor which is involved in brain development and myogenesis (Zhai et al., 2005). A recent gene expression study showed that the increase in mRNA levels of Aquaporin 9 (AQP9) and presence of $A Q P 9$ in blood could represent peripheral or central inflammatory events when accompanied with increase in levels of four other genes (Mesko et al., 2010). Annexin A3 (ANXA3) and Cysteine-rich, transmembrane (TM) module (CYSTM) are two other potential biomarkers for HD (Borovecki et al., 2005; Venancio and Aravind, 2010). ANXA3 is 
found to be up regulated in neuronal injury models and CYSTM is involved in stress response specifically heavy metal tolerance.

\section{Biochemical Markers}

Variability in clinical phenotype of HD and potential confounds of environmental and pharmacological factors, results in the use of combination of different biomarkers that might be efficient in tracking the progression of HD. Many potential biomarkers have been identified during the discovery of disrupted homoeostasis in HD. In a recent study, with the help of cross-sectional MRS the researchers have distinguished putaminal metabolites in premanifest and early HD individuals from controls (Sturrock et al., 2015). It was found that the total N-acetyl aspartate (tNAA) is lower in early HD and pre-manifest HD than in controls whereas the gliosis marker myo-inositol (MI) was robustly elevated in early HD. Another study, have also demonstrated metabolite changes in the caudate nucleus and putamen of HD gene carriers around disease onset (van Den Bogaard et al., 2014). These correlations of Total NAA with disease burden score suggest that this metabolite may be useful in identifying neurochemical responses to therapeutic agents.

Vasopressin has a role in fluid balance homoeostasis, increase in serum concentrations of vasopressin have been reported in HD (Wood et al., 2008). Increased concentrations of 8-hydroxy-2deoxyguanosine (8OHdG), an indicator of oxidative DNA injury, and increased concentrations of plasma lipid peroxide, lactic acid, 4-hydroxynoneal, and malondialdehyde in patients with HD make them a potential biomarker (Weir et al., 2011). Decrease in glutathione peroxidase and copper-zinc superoxide dismutase was observed in erythrocytes from HD patients compared with controls (Chen et al., 2007). Elevated cytokines levels including interleukins $4,6,8,10$, and 23 , TNF- $\alpha$, and clusterin have been identified in the post-mortem brain and plasma samples of patients with HD (Dalrymple et al., 2007). The inflammatory profile differences between control and gene carriers serve as potential biochemical marker for HD including rest of the above biomarkers. All these biomarkers would facilitate accurate evaluation of the effectiveness of new therapies and improve the safety and efficiency of clinical trials.

\section{References}

Alvarez-Erviti, L., Seow, Y., Schapira, A. H., Gardiner, C., Sargent, I. L., Wood, M. J., et al. (2011). Lysosomal dysfunction increases exosome-mediated alpha-synuclein release and transmission. Neurobiol. Dis. 42, 360-367. doi: 10.1016/j.nbd.2011.01.029

Aziz, N. A., Anguelova, G. V., Marinus, J., Lammers, G. J., and Roos, R. A. (2010). Sleep and circadian rhythm alterations correlate with depression and cognitive impairment in Huntington's disease. Parkinsonism Relat. Disord. 16, 345-350. doi: 10.1016/j.parkreldis.2010.02.009

Bekris, L. M., Yu, C. E., Bird, T. D., and Tsuang, D. W. (2010). Genetics of Alzheimer disease. J. Geriatr. Psychiatry Neurol. 23, 213-227. doi: 10.1177/0891988710383571

Berezovska, O., Ramdya, P., Skoch, J., Wolfe, M. S., Bacskai, B. J., and Hyman, B. T. (2003). Amyloid precursor protein associates with a nicastrindependent docking site on the presenilin 1-gamma-secretase complex in cells demonstrated by fluorescence lifetime imaging. J. Neurosci. 23, 4560-4566.

\section{Conclusion and Future Perspective}

In this short review, we have described current trends in the applications of molecular diagnostic techniques for early detection and diagnosis of neurodegenerative disorders focusing on $\mathrm{AD}, \mathrm{PD}, \mathrm{ALS}$, and $\mathrm{HD}$. We have discussed several biomolecular diagnostic markers that have been identified in the past decade that have an enormous scope for further research in the areas of both genetic and biochemical molecular markers. Biomolecular diagnostic markers may provide new insights regarding different diagnosis and therapeutic guidance to specific neurodegenerative diseases.

Molecular diagnostics for neurodegenerative diseases represent a multidisciplinary research area where a robust collaboration between neurologists, psychologists, biologist, and biomaterials scientists and other trained personnel with the necessary experience in managing the diseases is required. Future research directions might include designing and developing a combination of several biomolecular diagnostic markers for multifunctionalities. Such a multifunctional molecular diagnostic technology platform would significantly enhance the accuracy, specificity and sensitivity. Developing molecular diagnostics based on circulating miRNAs could also be a highly promising approach for developing minimally invasive screening tests for neurodegenerative disorders.

Future studies may also include developing a multicenter and prospective design of molecular diagnostics tools; measurement of multiple potential biomarkers and a prolonged clinical follow-up period (till death as end-point) that provide assessment of both clinical features and determinations of the biological diagnostics and eventually neuropathological confirmation by examining the brains of patients at death.

\section{Acknowledgments}

The authors wish to thank their respective parents for their support and encouragement.

Bogdanov, M., Brown, R. H., Matson, W., Smart, R., Hayden, D., O’Donnell, H., et al. (2000). Increased oxidative damage to DNA in ALS patients. Free Radic. Biol. Med. 29, 652-658. doi: 10.1016/S0891-5849(00)00349-X

Bokde, A. L. W., Meaney, J. F. M., Sheehy, N. P., Reilly, R. B., Abrahams, S., and Doherty, C. P. (2011). Advances in diagnostics for neurodegenerative disorders. Neurodegener. Dis. 2, 17-42. doi: 10.1007/978-1-84996-011-3_2

Bonifati, V., Rizzu, P., van Baren, M. J., Schaap, O., Breedveld, G. J., Krieger, E., et al. (2003). Mutations in the DJ-1 gene associated with autosomal recessive early-onset parkinsonism. Science 299, 256-259. doi: 10.1126/science.1077209

Borovecki, F., Lovrecic, L., Zhou, J., Jeong, H., Then, F., Rosas, H. D., et al. (2005). Genome-wide expression profiling of human blood reveals biomarkers for Huntington's disease. Proc. Natl. Acad. Sci. U.S.A. 102, 11023-11028. doi: 10.1073/pnas.0504921102

Calvo, A. C., Manzano, R., Mendonça, D. M., Muñoz, M. J., Zaragoza, P., and Osta, R. (2014). Amyotrophic lateral sclerosis: a focus on disease progression. Biomed Res. Int. 2014:925101. doi: 10.1155/2014/925101

Chen, C. M., Wu, Y. R., Cheng, M. L., Liu, J. L., Lee, Y. M., Lee, P. W., et al. (2007). Increased oxidative damage and mitochondrial abnormalities in the peripheral 
blood of Huntington's disease patients. Biochem. Biophys. Res. Commun. 359, 335-340. doi: 10.1016/j.bbrc.2007.05.093

Chung, S., Sonntag, K. C., Andersson, T., Bjorklund, L. M., Park, J. J., Kim, D. W., et al. (2002). Genetic engineering of mouse embryonic stem cells by Nurr1 enhances differentiation and maturation into dopaminergic neurons. Eur. J. Neurosci. 16, 1829-1838. doi: 10.1046/j.1460-9568.2002.02255.x

Ciurleo, R., Di Lorenzo, G., Bramanti, P., and Marino, S. (2014). Magnetic resonance spectroscopy: an in vivo molecular imaging biomarker for Parkinson's disease? Biomed Res. Int. 2014:519816. doi: 10.1155/2014/519816

Cova, E., Bongioanni, P., Cereda, C., Metelli, M. R., Salvaneschi, L., Bernuzzi, S., et al. (2010). Time course of oxidant markers and antioxidant defenses in subgroups of amyotrophic lateral sclerosis patients. Neurochem. Int. 56, 687-693. doi: 10.1016/j.neuint.2010.02.004

Dalrymple, A., Wild, E. J., Joubert, R., Sathasivam, K., Björkqvist, M., Petersén, A., et al. (2007). Proteomic profiling of plasma in Huntington's disease reveals neuroinflammatory activation and biomarker candidates. J. Proteome Res. 6 , 2833-2840. doi: 10.1021/pr0700753

DeCarli, C. (2003). Mild cognitive impairment: prevalence, prognosis, aetiology, and treatment. Lancet Neurol. 2, 15-21. doi: 10.1016/S1474-4422(03)00262-X

DeJesus-Hernandez, M., Mackenzie, I. R., Boeve, B. F., Boxer, A. L., Baker, M., Rutherford, N. J., et al. (2011). Expanded GGGGCC hexanucleotide repeat in noncoding region of C9ORF72 causes chromosome 9p-linked FTD and ALS. Neuron 72, 245-256. doi: 10.1016/j.neuron.2011.09.011

Dekker, M., Bonifati, V., van Swieten, J., Leenders, N., Galjaard, R. J., Snijders, P., et al. (2003). Clinical features and neuroimaging of PARK7-linked parkinsonism. Mov. Disord. 18, 751-757. doi: 10.1002/mds.10422

Duyckaerts, C., and Hauw, J. J. (2003). Lewy bodies, a misleading marker for Parkinson's disease? Bull. Acad. Natl. Med. 187, 277-292. discussion: 292-273.

el-Agnaf, O. M., and Irvine, G. B. (2002). Aggregation and neurotoxicity of alpha-synuclein and related peptides. Biochem. Soc. Trans. 30, 559-565. doi: $10.1042 /$ bst0300559

Fergani, A., Dupuis, L., Jokic, N., Larmet, Y., de Tapia, M., Rene, F., et al. (2005). Reticulons as markers of neurological diseases: focus on amyotrophic lateral sclerosis. Neurodegener. Dis. 2, 185-194. doi: 10.1159/0000 89624

Figueroa-Romero, C., Hur, J., Bender, D. E., Delaney, C. E., Cataldo, M. D., Smith, A. L., et al. (2012). Identification of epigenetically altered genes in sporadic amyotrophic lateral sclerosis. PLoS ONE 7:e52672. doi: 10.1371/journal.pone.0052672

Gasser, T., Bressman, S., Durr, A., Higgins, J., Klockgether, T., and Myers, R. H. (2003). State of the art review: molecular diagnosis of inherited movement disorders. Movement Disorders Society task force on molecular diagnosis. Mov. Disord. 18, 3-18. doi: 10.1002/mds. 10338

Gasser, T., Dichgans, M., Finsterer, J., Hausmanowa-Petrusewicz, I., Jurkat-Rott, K., Klopstock, T., et al. (2001a). EFNS Task Force on Molecular Diagnosis of Neurologic Disorders: guidelines for the molecular diagnosis of inherited neurologic diseases. First of two parts. Eur. J. Neurol. 8, 299-314. doi: 10.1046/j.1468-1331.2001.00226.x

Gasser, T., Dichgans, M., Finsterer, J., Hausmanowa-Petrusewicz, I., Jurkat-Rott, K., Klopstock, T., et al. (2001b). EFNS Task Force on Molecular Diagnosis of Neurologic Disorders: guidelines for the molecular diagnosis of inherited neurologic diseases. Second of two parts. Eur. J. Neurol. 8, 407-424. doi: 10.1046/j.1468-1331.2001.00228.x

Goodall, E. F., Heath, P. R., Bandmann, O., Kirby, J., and Shaw, P. J. (2013). Neuronal dark matter: the emerging role of microRNAs in neurodegeneration. Front. Cell. Neurosci. 7:178. doi: 10.3389/fncel.2013.00178

Grasso, M., Piscopo, P., Confaloni, A., and Denti, M. A. (2014). Circulating miRNAs as biomarkers for neurodegenerative disorders. Molecules 19, 6891-6910. doi: 10.3390/molecules19056891

Hardy, J., and Selkoe, D. J. (2002). The amyloid hypothesis of Alzheimer's disease: progress and problems on the road to therapeutics. Science 297, 353-356. doi: $10.1126 /$ science. 1072994

Harvey, P. D. (2012). Clinical applications of neuropsychological assessment. Dialogues Clin. Neurosci. 14, 91-99.

Hu, Y., Chopra, V., Chopra, R., Locascio, J. J., Liao, Z., Ding, H., et al. (2011). Transcriptional modulator H2A histone family, member Y (H2AFY) marks Huntington disease activity in man and mouse. Proc. Natl. Acad. Sci. U.S.A. 108, 17141-17146. doi: 10.1073/pnas.1104409108
Jankovic, J., Rajput, A. H., McDermott, M. P., and Perl, D. P. (2000). The evolution of diagnosis in early Parkinson disease. Parkinson Study Group. Arch. Neurol. 57, 369-372. doi: 10.1001/archneur.57.3.369

Joyce, N. C., and Carter, G. T. (2013). Electrodiagnosis in persons with amyotrophic lateral sclerosis. PM R 5, S89-S95. doi: 10.1016/j.pmrj.2013.03.020

Lachenal, G., Pernet-Gallay, K., Chivet, M., Hemming, F. J., Belly, A., Bodon, G., et al. (2011). Release of exosomes from differentiated neurons and its regulation by synaptic glutamatergic activity. Mol. Cell. Neurosci. 46, 409-418. doi: 10.1016/j.mcn.2010.11.004

Leroy, E., Boyer, R., Auburger, G., Leube, B., Ulm, G., Mezey, E., et al. (1998). The ubiquitin pathway in Parkinson's disease. Nature 395, 451-452. doi: $10.1038 / 26652$

Lin, J., Li, J., Huang, B., Liu, J., Chen, X., Chen, X. M., et al. (2015). Exosomes: novel biomarkers for clinical diagnosis. Scientific World Journal 2015:657086. doi: $10.1155 / 2015 / 657086$

Loane, C., and Politis, M. (2011). Positron emission tomography neuroimaging in Parkinson's disease. Am. J. Transl. Res. 3, 323-341.

Lovrecic, L., Kastrin, A., Kobal, J., Pirtosek, Z., Krainc, D., and Peterlin, B. (2009). Gene expression changes in blood as a putative biomarker for Huntington's disease. Mov. Disord. 24, 2277-2281. doi: 10.1002/mds.22477

Mapstone, M., Cheema, A. K., Fiandaca, M. S., Zhong, X., Mhyre, T. R., Macarthur, L. H., et al. (2014). Plasma phospholipids identify antecedent memory impairment in older adults. Nat. Med. 20, 415-418. doi: 10.1038/nm.3466

Marek, K., Seibyl, J., and Holloway, R. (2000). A multicenter assessment of dopamine transporter imaging with DOPASCAN/SPECT in parkinsonism. Parkinson Study Group. Neurology 55, 1540-1547. doi: 10.1212/WNL.55.10.1540

Margis, R., Margis, R., and Rieder, C. R. (2011). Identification of blood microRNAs associated to Parkinsonis disease. J. Biotechnol. 152, 96-101. doi: 10.1016/j.jbiotec.2011.01.023

Martin, L. J., and Wong, M. (2013). Aberrant regulation of DNA methylation in amyotrophic lateral sclerosis: a new target of disease mechanisms. Neurotherapeutics 10, 722-733. doi: 10.1007/s13311-013-0205-6

Mastrokolias, A., Ariyurek, Y., Goeman, J. J., van Duijn, E., Roos, R. A., van Der Mast, R. C., et al. (2015). Huntington's disease biomarker progression profile identified by transcriptome sequencing in peripheral blood. Eur. J. Hum. Genet. doi: 10.1038/ejhg.2014.281. [Epub ahead of print].

Mesko, B., Poliska, S., Szegedi, A., Szekanecz, Z., Palatka, K., Papp, M., et al. (2010). Peripheral blood gene expression patterns discriminate among chronic inflammatory diseases and healthy controls and identify novel targets. $B M C$ Med. Genomics 3:15. doi: 10.1186/1755-8794-3-15

Miller, D. B., and O'Callaghan, J. P. (2015). Biomarkers of Parkinson's disease: present and future. Metabolism 64, S40-S46. doi: 10.1016/j.metabol.2014.10.030

Molochnikov, L., Rabey, J. M., Dobronevsky, E., Bonucelli, U., Ceravolo, R., Frosini, D., et al. (2012). A molecular signature in blood identifies early Parkinson's disease. Mol. Neurodegener. 7:26. doi: 10.1186/1750-13 26-7-26

Pankratz, N., Nichols, W. C., Uniacke, S. K., Halter, C., Rudolph, A., Shults, C., et al. (2003). Significant linkage of Parkinson disease to chromosome 2q36-37. Am. J. Hum. Genet. 72, 1053-1057. doi: 10.1086/374383

Peterson, D. A. (2002). Stem cells in brain plasticity and repair. Curr. Opin. Pharmacol. 2, 34-42. doi: 10.1016/S1471-4892(01)00118-7

Philips, T., and Robberecht, W. (2011). Neuroinflammation in amyotrophic lateral sclerosis: role of glial activation in motor neuron disease. Lancet Neurol. 10, 253-263. doi: 10.1016/S1474-4422(11)70015-1

Posner, H. B., Cano, S., Carrillo, M. C., Selnes, O., Stern, Y., Thomas, R. G., et al. (2013). Establishing the psychometric underpinning of cognition measures for clinical trials of Alzheimer's disease and its precursors: a new approach. Alzheimers Dement. 9, S56-S60. doi: 10.1016/j.jalz.2012. 10.008

Poulopoulou, C., Davaki, P., Koliaraki, V., Kolovou, D., Markakis, I., and Vassilopoulos, D. (2005). Reduced expression of metabotropic glutamate receptor 2mRNA in T cells of ALS patients. Ann. Neurol. 58, 946-949. doi: 10.1002/ana.20675

Rachakonda, V., Pan, T. H., and LE, W. D. (2004). Biomarkers of neurodegenerative disorders: how good are they? Cell Res. 14, 347-358. doi: $10.1038 /$ sj.cr.7290235 
Rajendran, L., Honsho, M., Zahn, T. R., Keller, P., Geiger, K. D., Verkade, P., et al. (2006). Alzheimer's disease beta-amyloid peptides are released in association with exosomes. Proc. Natl. Acad. Sci. U.S.A. 103, 11172-11177. doi: $10.1073 /$ pnas. 0603838103

Robelin, L., and Gonzalez De Aguilar, J. L. (2014). Blood biomarkers for amyotrophic lateral sclerosis: myth or reality? Biomed Res. Int. 2014:525097. doi: $10.1155 / 2014 / 525097$

Runne, H., Regulier, E., Kuhn, A., Zala, D., Gokce, O., Perrin, V., et al. (2008). Dysregulation of gene expression in primary neuron models of Huntington's disease shows that polyglutamine-related effects on the striatal transcriptome may not be dependent on brain circuitry. J. Neurosci. 28, 9723-9731. doi: 10.1523/JNEUROSCI.3044-08.2008

Russo, I., Bubacco, L., and Greggio, E. (2012). Exosomes-associated neurodegeneration and progression of Parkinson's disease. Am. J. Neurodegener. Dis. 1, 217-225.

Sair, H. I., Doraiswamy, P. M., and Petrella, J. R. (2004). In vivo amyloid imaging in Alzheimer's disease. Neuroradiology 46, 93-104. doi: 10.1007/s00234-0031034-9

Saman, S., Kim, W., Raya, M., Visnick, Y., Miro, S., Saman, S., et al. (2012). Exosome-associated tau is secreted in tauopathy models and is selectively phosphorylated in cerebrospinal fluid in early Alzheimer disease. J. Biol. Chem. 287, 3842-3849. doi: 10.1074/jbc.M111.277061

Sheinerman, K. S., Tsivinsky, V. G., Crawford, F., Mullan, M. J., Abdullah, L., and Umansky, S. R. (2012). Plasma microRNA biomarkers for detection of mild cognitive impairment. Aging. 4, 590-605.

Shinde, S., Mukhopadhyay, S., Mohsen, G., and Khoo, S. K. (2015). Biofluid-based microRNA biomarkers for Parkinsons disease: an overview and update. AIMS Med. Sci. 2, 15-25. doi: 10.3934/medsci.2015.1.15

Shinto, A. S., Antony, J., Kamaleshwaran, K., Vijayan, K., Selvan, A., Korde, A., et al. (2014). Correlative $(99 \mathrm{~m}) \mathrm{tc}$-labeled tropane derivative single photon emission computer tomography and clinical assessment in the staging of Parkinson disease. World J. Nucl. Med. 13, 178-183. doi: 10.4103/14501147.144818

Sjögren, M., Andreasen, N., and Blennow, K. (2003). Advances in the detection of Alzheimer's disease-use of cerebrospinal fluid biomarkers. Clin. Chim. Acta 332, 1-10. doi: 10.1016/S0009-8981(03)00121-9

Soreq, L., Salomonis, N., Bronstein, M., Greenberg, D. S., Israel, Z., Bergman, H., et al. (2013). Small RNA sequencing-microarray analyses in Parkinson leukocytes reveal deep brain stimulation-induced splicing changes that classify brain region transcriptomes. Front. Mol. Neurosci. 6:10. doi: 10.3389/fnmol.2013.00010

Sturrock, A., Laule, C., Wyper, K., Milner, R. A., Decolongon, J., Dar Santos, R., et al. (2015). A longitudinal study of magnetic resonance spectroscopy Huntington's disease biomarkers. Mov. Disord. 30, 393-401. doi: $10.1002 / \mathrm{mds} .26118$

Sunderland, T., Linker, G., Mirza, N., Putnam, K. T., Friedman, D. L., Kimmel, L. H., et al. (2003). Decreased beta-amyloid1-42 and increased tau levels in cerebrospinal fluid of patients with Alzheimer disease. JAMA 289, 2094-2103. doi: 10.1001/jama.289.16.2094

Tissingh, G., Bergmans, P., Booij, J., Winogrodzka, A., Stoof, J. C., Wolters, E. C., et al. (1997). [123I]beta-CIT single-photon emission tomography in
Parkinson's disease reveals a smaller decline in dopamine transporters with age than in controls. Eur. J. Nucl. Med. 24, 1171-1174. doi: 10.1007/BF012 54252

Torrent, R., De Angelis Rigotti, F., Dell'era, P., Memo, M., Raya, A., and Consiglio, A. (2015). Using iPS cells toward the understanding of Parkinson's disease. J. Clin. Med. 4, 548-566. doi: 10.3390/jcm 4040548

Valente, E. M., Abou-Sleiman, P. M., Caputo, V., Muqit, M. M., Harvey, K., Gispert, S., et al. (2004). Hereditary early-onset Parkinson's disease caused by mutations in PINK1. Science 304, 1158-1160. doi: 10.1126/science.10 96284

van Den Bogaard, S. J., Dumas, E. M., Teeuwisse, W. M., Kan, H. E., Webb, A., van Buchem, M. A., et al. (2014). Longitudinal metabolite changes in Huntington's disease during disease onset. J. Huntingtons. Dis. 3, 377-386. doi: 10.3233/JHD-140117

Venancio, T. M., and Aravind, L. (2010). CYSTM, a novel cysteine-rich transmembrane module with a role in stress tolerance across eukaryotes. Bioinformatics 26, 149-152. doi: 10.1093/bioinformatics/btp647

Weir, D. W., Sturrock, A., and Leavitt, B. R. (2011). Development of biomarkers for Huntington's disease. Lancet Neurol. 10, 573-590. doi: 10.1016/S14744422(11)70070-9

Wild, E. J., Boggio, R., Langbehn, D., Robertson, N., Haider, S., Miller, J. R., et al. (2015). Quantification of mutant huntingtin protein in cerebrospinal fluid from Huntington's disease patients. J. Clin. Invest. 125, 1979-1986. doi: 10.1172/JCI80743

Wood, N. I., Goodman, A. O., van der Burg, J. M., Gazeau, V., Brundin, P., Björkqvist, M., et al. (2008). Increased thirst and drinking in Huntington's disease and the R6/2 mouse. Brain Res. Bull. 76, 70-79. doi: 10.1016/j.brainresbull.2007.12.007

Wurtman, R. (2015). Biomarkers in the diagnosis and management of Alzheimer's disease. Metabolism 64, S47-S50. doi: 10.1016/j.metabol.2014.10.034

Xi, Y., Nakajima, G., Gavin, E., Morris, C. G., Kudo, K., Hayashi, K., et al. (2007). Systematic analysis of microRNA expression of RNA extracted from fresh frozen and formalin-fixed paraffin-embedded samples. RNA 13, 1668-1674. doi: $10.1261 /$ rna.642907

Young, A. B. (2009). Four decades of neurodegenerative disease research: how far we have come! J. Neurosci. 29, 12722-12728. doi: 10.1523/JNEUROSCI.376709.2009

Zhai, W., Jeong, H., Cui, L., Krainc, D., and Tjian, R. (2005). In vitro analysis of huntingtin-mediated transcriptional repression reveals multiple transcription factor targets. Cell 123, 1241-1253. doi: 10.1016/j.cell.2005.10.030

Conflict of Interest Statement: The authors declare that the research was conducted in the absence of any commercial or financial relationships that could be construed as a potential conflict of interest.

Copyright $\odot 2015$ Agrawal and Biswas. This is an open-access article distributed under the terms of the Creative Commons Attribution License (CC BY). The use, distribution or reproduction in other forums is permitted, provided the original author(s) or licensor are credited and that the original publication in this journal is cited, in accordance with accepted academic practice. No use, distribution or reproduction is permitted which does not comply with these terms. 